\section{0-010 INFLUENCE OF THROMBECTOMY VOLUME ON NON- PHYSICIAN STAFF BURNOUT AND ATTRITION IN NEUROINTERVENTIONAL TEAMS}

${ }^{1} \mathrm{~K}$ Fargen, ${ }^{2} \mathrm{~S}$ Ansari, ${ }^{3} \mathrm{~A}$ Spiotta, ${ }^{4} \mathrm{G}$ Dabus, ${ }^{5} \mathrm{M}$ Mokin, ${ }^{1} \mathrm{P}$ Brown ${ }^{*},{ }^{1} \mathrm{~S}$ Wolfe, ${ }^{1} \mathrm{C}$ Kittel, ${ }^{6} \mathrm{P}$ Kan, ${ }^{7} \mathrm{~B}$ Baxter, ${ }^{8} \mathrm{R}$ de Leacy, ${ }^{9} \mathrm{~J}$ Milburn, ${ }^{10} \mathrm{~S}$ Munich, ${ }^{11} \mathrm{~A}$ Ducruet, ${ }^{12} \mathrm{~A}$ Reeves, ${ }^{13} \mathrm{~J}$ Fraser, ${ }^{14} \mathrm{R}$ Starke, ${ }^{15} \mathrm{~A}$ Jadhav, ${ }^{16} \mathrm{~W}$ Mack, ${ }^{17} \mathrm{~A}$ Arthur, ${ }^{18} \mathrm{G}$ Pride, ${ }^{19} \mathrm{~S}$ Sheth, ${ }^{20} \mathrm{~T}$ Leslie-Mazwi, ${ }^{21} \mathrm{~J}$ Hirsch. ${ }^{1}$ Neurological Surgery and Radiology, Wake Forest Baptist University, WinstonSalem, NC; ${ }^{2}$ Radiology, Neurology, Neurological Surgery, Northwestern University, Chicago, $\mathrm{IL}_{i}{ }^{3}$ Neurosurgery, Medical University of South Carolina, Charleston, SC; ${ }^{4}$ Miami Cardiac and Vascular Institute and Baptist Neuroscience Center, Miami, FL; ${ }^{5}$ Neurology and Neurosurgery, University of South Florida, Tampa, FL; ${ }^{6}$ Neurosurgery, Baylor College of Medicine, Houston, TX; ${ }^{7}$ Radiology, Lehigh Valley Health Network, Allentown, PA; ${ }^{8}$ Neurosurgery and Radiology, Mount Sinai Hospital, New York, NY; ${ }^{9}$ Radiology, Ochnsner Medical Center, New Orleans, LA; ${ }^{10}$ Neurosurgery, Rush University, Chicago, IL: ${ }^{11}$ Neurosurgery, Barrow Neurological Institute, Phoenix, AZ; ${ }^{12}$ Radiology, Neuroendovascular Division, University of Kansas Medical Center, Kansas City, KS; ${ }^{13}$ Neurosurgery, Neurology, Radiology and Neuroscience, University of Kentucky, Lexington, $\mathrm{KY} ;{ }^{14}$ Neurosurgery and Neuroradiology, University of Miami and Jackson Memorial Hospital, Miami, FL; ${ }^{15}$ Neurology, University of Pittsburgh Medical Center, Pittsburgh, PA; ${ }^{16}$ Neurosurgery, University of Southern California, Los Angeles, CA; ${ }^{17}$ Neurosurgery, University of Tennessee-Semmes Murphey Clinic, Memphis, $T_{N} ;{ }^{18}$ Neurosurgery, University of Texas-Southwestern, Dallas, TX; ${ }^{19}$ Neurology, University of Texas Medical Center, Houston, TX; ${ }^{20}$ Radiology, West Virginia University, Morgantown, WW: ${ }^{21}$ Interventional Neuroradiology, Massachusetts General Hospital, Boston, MA

\subsection{6/neurintsurg-2020-SNIS. 10}

Introduction Burnout takes a heavy toll on healthcare providers. We sought to assess the prevalence and risk factors for burnout among neurointerventional (NI) non-physician procedural staff (nurses and technologists) given increasing thrombectomy demands.

Materials and Methods A 41 question online survey containing questions including the Maslach Burnout Inventory-Human Services Survey for Medical Personnel was distributed to NI nurses and radiology technologists at 20 U.S. endovascular capable stroke centers.

Results 244 responses were received (64\% response rate). Median (inter-quartile range) composite scores for emotional exhaustion were 25 (15-35), depersonalization 6 (2-11) and personal accomplishment 39 (35-43). Fifty-one percent of respondents met established criteria for burnout. There was no significant relationship between hospital thrombectomy volume, call frequency, call cases covered, or length of commute. On multiple logistic regression analysis, feeling underappreciated by hospital leadership (OR 4.1; $\mathrm{P}<0.001)$ and working with difficult/unpleasant physicians (OR 1.2; $\mathrm{P}=0.05$ ) were strongly associated with burnout. At participating centers, nurse and technologist attrition was 25\% over the last year. Over 50\% of respondents indicated they had strongly considered leaving their position over the last 2 years.

\begin{tabular}{|c|c|c|c|}
\hline $\begin{array}{l}\text { Multiple Logistic Regression } \\
\text { Outcome: Burnout Classification }\end{array}$ & Coefficient & Odds Ratio $[95 \% \mathrm{Cl}]$ & P-value \\
\hline Happiness in Career Choice & -0.59 & $0.55[0.43-0.69]$ & $<0.001$ \\
\hline $\begin{array}{l}\text { Feeling Under-Appreciated by } \\
\text { Hospital/Department }\end{array}$ & 1.4 & $4.10[2.07-8.31]$ & $<0.001$ \\
\hline $\begin{array}{l}\text { Physicians More Difficult to Work } \\
\text { With }\end{array}$ & 0.19 & $1.21[1.00-1.48]$ & 0.05 \\
\hline $\begin{array}{l}\text { Increasing Effect of Work on } \\
\text { Family Life }\end{array}$ & 0.13 & $1.14[1.01-1.30]$ & 0.04 \\
\hline
\end{tabular}

Conclusion This survey of United States NI non-physician procedural staff demonstrates a self-reported burnout prevalence of $51 \%$. This was driven more by interaction with leadership and physician staff than by thrombectomy procedural volume and stroke call. Attrition among NI non-physician procedural staff is high.

Disclosures K. Fargen: None. S. Ansari: None. A. Spiotta: None. G. Dabus: None. M. Mokin: None. P. Brown: None. S. Wolfe: None. C. Kittel: None. P. Kan: None. B. Baxter: None. R. de Leacy: None. J. Milburn: None. S. Munich: None. A. Ducruet: None. A. Reeves: None. J. Fraser: None. R. Starke: None. A. Jadhav: None. W. Mack: None. A. Arthur: None. G. Pride: None. S. Sheth: None. T. LeslieMazwi: None. J. Hirsch: None.

\section{0-011 DRIVERS OF VARIATION IN 90-DAY EPISODE PAYMENTS AFTER MECHANICAL THROMBECTOMY FOR ACUTE ISCHEMIC STROKE}

B Daou*, M Yost, J Syrjamaki, K Fearer, S Koduri, J Burke, J Gemmete, N Chaudhary, B Thompson, A Pandey. Neurosurgery, University of Michigan, Ann Arbor, MI

10.1136/neurintsurg-2020-SNIS. 11

Introduction Although mechanical thrombectomy for acute ischemic stroke from a large vessel occlusion is now the standard of care, little is known about cost variations in stroke patients following thrombectomy.

Methods We evaluated claims data for thrombectomy-performing hospitals within Michigan through a registry that includes detailed episode payment information for both Medicare and privately insured patients. We aimed to

Abstract 0-011 Table 1 Sample characteristics for terciles of hospital's thrombectomy spending. P values from ANOVA and Chi Squared tests

\begin{tabular}{|c|c|c|c|c|}
\hline & \multicolumn{4}{|c|}{ Hospital Thrombectomy Spending Tercile } \\
\hline & Low $(\mathrm{N}=218)$ & $\begin{array}{l}\text { Medium } \\
(\mathrm{N}=477)\end{array}$ & High $(\mathrm{N}=381)$ & P-value \\
\hline Hospitals, n & 5 & 6 & 5 & \\
\hline Age, mean $\pm S D$ & $74.7 \pm 12.7$ & $74.8 \pm 13.0$ & $73.6 \pm 12.4$ & 0.3429 \\
\hline Male, $n(\%)$ & $81(37.2)$ & $216(45.3)$ & $180(47.2)$ & 0.0490 \\
\hline $\begin{array}{l}\text { High 6-month prior } \\
\text { spending, } \mathrm{n}(\%)\end{array}$ & $101(46.3)$ & $230(48.2)$ & $170(44.6)$ & 0.5746 \\
\hline \multicolumn{5}{|l|}{ Payer, n (\%) } \\
\hline Medicare FFS/Medicare & $166(76.2)$ & $359(75.3)$ & $285(74.8)$ & 0.9349 \\
\hline \multicolumn{5}{|l|}{ Advantage } \\
\hline$B C B S M P P O$ and $B C N$ & $52(23.9)$ & $118(24.7)$ & $96(25.2)$ & \\
\hline $\begin{array}{l}\text { Mean Count of HCCs per } \\
\text { patient, mean } \pm S D\end{array}$ & $5.9 \pm 3.3$ & $6.0 \pm 3.0$ & $6.0 \pm 3.3$ & 0.9372 \\
\hline \multicolumn{5}{|c|}{ Major HCC Categories, n (\%) } \\
\hline Chronic Kidney Disease & $12(5.5)$ & $11(2.3)$ & $20(5.25)$ & 0.0407 \\
\hline Cancer & $29(13.3)$ & $64(13.4)$ & $47(12.3)$ & 0.8872 \\
\hline COPD & $39(17.9)$ & $116(24.3)$ & $85(22.3)$ & 0.1679 \\
\hline $\mathrm{CHF}$ & $84(38.5)$ & $166(34.8)$ & $116(30.6)$ & 0.1179 \\
\hline Diabetes & $80(36.7)$ & $175(36.7)$ & $132(34.7)$ & 0.7998 \\
\hline Vascular Disease & $50(22.9)$ & $132(27.7)$ & $117(30.7)$ & 0.1236 \\
\hline Respiratory Dysfunction & $18(8.3)$ & $37(7.8)$ & $22(5.8)$ & 0.4165 \\
\hline Neurologic Disorders & $183(83.9)$ & $404(84.7)$ & $315(82.7)$ & 0.7263 \\
\hline
\end{tabular}


Abstract 0-011 Table 2 Post discharge subcomponent spending across hospital terciles

\begin{tabular}{|c|c|c|c|c|}
\hline & \multicolumn{4}{|c|}{ Hospital Thrombectomy Spending Tercile } \\
\hline & $\begin{array}{l}\text { Low } \\
(\mathrm{N}=218)\end{array}$ & $\begin{array}{l}\text { Medium } \\
(\mathrm{N}=477)\end{array}$ & $\begin{array}{l}\text { High } \\
(\mathrm{N}=381)\end{array}$ & P-value \\
\hline $\begin{array}{l}\text { SNF spending, } \\
\text { mean } \pm \text { SD }\end{array}$ & $\begin{array}{l}\$ 5,570 \pm \\
\$ 11,790\end{array}$ & $\begin{array}{l}\$ 7,201 \pm \\
\$ 15,883\end{array}$ & $\begin{array}{l}\$ 8,738 \pm \\
\$ 17,738\end{array}$ & 0.0585 \\
\hline SNF utilization, $n(\%)$ & $35(16.1)$ & $104(21.8)$ & $118(31.0)$ & $<0.0001$ \\
\hline $\begin{array}{l}\text { SNF spending when } \\
\text { present, mean } \pm \text { SD }\end{array}$ & $\begin{array}{l}\$ 18,396 \pm \\
\$ 14,981\end{array}$ & $\begin{array}{l}\$ 21,468 \pm \\
\$ 21,142\end{array}$ & $\begin{array}{l}\$ 23,445 \pm \\
\$ 22,375\end{array}$ & 0.2591 \\
\hline $\begin{array}{l}\text { Home health spending, } \\
\text { mean } \pm \text { SD }\end{array}$ & $\begin{array}{l}\$ 903 \pm \\
\$ 3,128\end{array}$ & $\begin{array}{l}\$ 1,720 \pm \\
\$ 5,492\end{array}$ & $\begin{array}{l}\$ 1,760 \pm \\
\$ 5,556\end{array}$ & 0.0998 \\
\hline Home health utilization, $n(\%)$ & $55(25.2)$ & $122(25.6)$ & $108(28.4)$ & 0.5896 \\
\hline $\begin{array}{l}\text { Home health spending } \\
\text { when present, mean } \pm \text { SD }\end{array}$ & $\begin{array}{l}\$ 3,580 \pm \\
\$ 5,437\end{array}$ & $\begin{array}{l}\$ 6,727 \pm \\
\$ 9,202\end{array}$ & $\begin{array}{l}\$ 6,208 \pm \\
\$ 9,041\end{array}$ & 0.0712 \\
\hline $\begin{array}{l}\text { OP rehab spending, } \\
\text { mean }(S D)\end{array}$ & $\begin{array}{l}\$ 4,655 \pm \\
\$ 8,068\end{array}$ & $\begin{array}{l}\$ 4,828 \pm \\
\$ 8,725\end{array}$ & $\begin{array}{l}\$ 4,557 \pm \\
\$ 8,221\end{array}$ & 0.8933 \\
\hline OP rehab utilization, $n(\%)$ & $85(39.0)$ & $190(39.8)$ & $164(43.3)$ & 0.4802 \\
\hline $\begin{array}{l}\text { OP rehab spending when } \\
\text { present, mean } \pm S D\end{array}$ & $\begin{array}{l}\$ 11,939 \pm \\
\$ 8,953)\end{array}$ & $\begin{array}{l}\$ 12,121 \pm \\
\$ 10,143\end{array}$ & $\begin{array}{l}\$ 10,523 \pm \\
\$ 9,667\end{array}$ & 0.2735 \\
\hline $\begin{array}{l}\text { Other OP spending, } \\
\text { mean (SD) }\end{array}$ & $\begin{array}{l}\$ 1,245 \pm \\
\$ 3,294\end{array}$ & $\begin{array}{l}\$ 928 \pm \\
\$ 2,333\end{array}$ & $\begin{array}{l}\$ 943 \pm \\
\$ 2,255\end{array}$ & 0.2721 \\
\hline $\begin{array}{l}\text { Other OP spending } \\
\text { utilization, } \\
\mathrm{n}(\%)\end{array}$ & $101(46.3)$ & $263(55.1)$ & $189(49.6)$ & 0.0672 \\
\hline $\begin{array}{l}\text { Other OP spending when } \\
\text { present, mean } \pm \text { SD }\end{array}$ & $\begin{array}{l}\$ 2,686 \pm \\
\$ 4,432\end{array}$ & $\begin{array}{l}\$ 1,684 \pm \\
\$ 2,935\end{array}$ & $\begin{array}{l}\$ 1,902 \pm \\
\$ 2,906\end{array}$ & 0.0311 \\
\hline
\end{tabular}

analyze price-standardized and risk-adjusted 90-day episode payments in patients who underwent thrombectomy at Michigan hospitals. Hospitals were grouped into three payment terciles for comparison. Statistical analysis was carried out using unpaired t-test, Chi-square and ANOVA tests as appropriate. Results: 1,076 thrombectomy cases treated at 16 centers were analyzed. The average 90-day episode payment by hospital ranged from $\$ 53,046$ to $\$ 81,767$, with a mean of $\$ 65,357$. A $\$ 20,467$ difference $(35.1 \%)$ existed between the high and low payment hospital terciles $(\mathrm{P}<0.0001)$. The primary drivers of payment variation were related to post-discharge care which accounted for $38 \%$ of the payment variation $(\mathrm{P}=0.0058$, inter-tercile range $\$ 11,977-\$ 19,703)$ and readmissions accounting for $26 \%(\mathrm{P}=0.016$, inter-tercile range $\$ 3,315$ $\$ 7,992)$. This was followed by professional payments representing $20 \%$ of the variation $(\mathrm{P}<0.0001$, inter-tercile range 7,525 to $\$ 9,922$ ), while index hospitalization payment was responsible for only $16 \%$ of the 90 -day episode payment variation $(\mathrm{P}=0.10$, inter-tercile range $\$ 35,432$ $\$ 41,099$ ). High payment hospitals were more likely to discharge to a skilled nursing facility (difference of $14.9 \%$, $\mathrm{P}<0.0001)$.

Conclusion There is a wide variation in 90-day episode payments for patients undergoing thrombectomy across centers within a single state.

Disclosures B. Daou: None. M. Yost: None. J. Syrjamaki: None. K. Fearer: None. S. Koduri: None. J. Burke: None. J. Gemmete: None. N. Chaudhary: None. B. Thompson: None. A. Pandey: None. 